\title{
Ein kleiner Beitrag \\ zur Kenntnis der Ornis der Provinz Sachsen.
}

\section{Von W. Grafsmann.}

Als Landmann der tagtäglich vom frühen Morgen bis zum späten Abend in Gottes freier Natur herumstreift und offenes Auge und $\mathrm{Ohr}$ für die Vogelwelt hat, will ich im Nachfolgenden einen kleinen Beitrag zur Verbreitung der Kenntnis der Vögel der Provinz Sachsen liefern.

Mein Beobachtungsbezirk umfafst mit einem Gesamtareal von 420 ha den Guts- und Gemeindebezirk Grauwinkel b. Schönwalde, dieses liegt im nordöstlichen Teil der Provinz Sachsen, hart an der brandenburgischen Grenze. Das Gelände ist eben, aber abwechslungsreich, der Boden sehr leicht, so dafs nur im Wiesengürtel und an den zahlreichen Grabenrändern üppiger Pflanzenwuchs vorhanden ist. Von der Gesamtfläche entfallen $1000 \mathrm{Mrg}$. auf Ackerland, $230 \mathrm{Mrg}$. auf Wiesen u. Gärten und $450 \mathrm{Mrg}$. auf Kiefernwald, hiervon $150 \mathrm{Mrg}$. junge Anpflanzungen.

Aufserdem ist ein schöner, alter, 15 Mrg. grofser Park hier, mit viel Unterholz und Gebüsch.

Bei der folgenden Aufzählung habe ich nur die Brutvögel meines oben bestimmten Beobachtungsbezirks numeriert.

1. Erithacus luscinia L. Am 25. 4. vorm. hörte ich die Nachtigall zum ersten Male, bald darauf hörte ich einige Tage lang noch ein 2. Männchen, das dann aber bald weiterzog, die zurückbleibende Nachtigall sang sehr fleifsig, ich hörte sie während des ganzen Mai fast zu jeder Tages- und Nachtzeit, nach längerer Pause sang sie noch am 28. 6. einige kurze Strophen. Sie brütete im Parke.

2. Erithacus rubeculus L. Nur e in Paar nistete im Parke.

3. Ruticilla titys (L.). Im Dorfe 3 Brutpaare, benutzten in 2 Fällen die Gerüstlöcher der Gebäude als Niststätte.

4. Pratincola rubetra L. Ich beobachtete 3 Paare und zwar sämtlich in einer jungen Erlenanpflanzung mit üppigem Graswuchs. Auf den grofsen Wiesen habe ich den Wiesenschmätzer nur vorübergehend gesehen, der Mangel an Gesträuch behagte ihm wohl nicht!

5. Saxicola oenante L. Es brüteten 2 Paare in meinem Gebiet, das eine Paar in der Nähe einer Kiesgrube, das andere in einem Steinhaufen in einer einjährigen Kiefernanpflanzung und zwar, trotzdem dieser ca. $80 \mathrm{~cm}$ hohe Steinhaufen häufig der Späheplatz von Krähen, Bussard und Turmfalke war. Obwohl ich in dem Steinhaufen das Nest vermutete, konnte ich es zunächst doch nicht ausfindig machen, bis ich endlich durch das Gezirpe der 
Jungen überführt wurde. Das Nest befand sich ziemlich in der Mitte unter einigen grölseren des sonst nur aus faustgrofsen Steinen bestehenden Haufens, es schien mir kaum glaublich, wie die Vögel sich durch die schmalen Ritzen hindurchwinden konnten. Den spähenden Krähen und Bussarden mag wohl der Schnabel wässerig geworden sein bei dem verlockenden Gezirpe.

Die alten Steinschmätzer waren auch über derartige ungebetene Besuche garnicht so empört und erregt wie das sonst der Fall zu sein pflegt, sie schienen eben von der Uneinnehmbarkeit ihrer Festung überzeugt zu sein. Ich habe dieses gar oft von dem ca. $200 \mathrm{~m}$ entfernten Hochsitze mit meinem ZeifsTrieder beobachtet.

6. Turdus musicus L. Ein Paar brütete im Park auf einer jungen Buche in etwa $2 \frac{1}{2} \mathrm{~m}$ Höhe, am 4. 6. waren Eier und Junge im Neste. Eine 2. Singdrossel hörte ich allabendlich am Rande einer Kiefernschonung.

7. Turdus pilaris L. Den ganzen Frühling bindurch bis jetzt zum Juli trieben sich die Wacholderdrosseln in Scharen bis zu 20 St. hier herum, gebrütet haben nur 3-4 Paar, alle in einem Kiefernstangenholze. Ende Mai fand ich ein zerstörtes Nest mit Eischalen, jedenfalls war ein Eichhörnchen der Räuber gewesen. Die Nester waren, bis auf eins, im Wipfel der Bäume, das erstere stand in einer Astgabel in halber Höhe am Stamme. Im grofsen Ganzen ist die Wachholderdrossel auch hier ein echter Zigeuner, der überall und nirgends ist.

8. Turdus merula L. Im Parke waren 6 Brutpaare, 3 im Walde in Kiefernschonungen. Bereits am 21. 4. fand ich ein Nest mit halbflüggen Jungen; im Walde stand ein Nest in $4 \mathrm{~m}$ Höhe auf einer Kiefer. Alle Nester, die ich bisher entdeckt habe und deren Zahl mit 40 nicht $\mathrm{zu}$ hoch angegeben ist, enthielten nie mehr als 5 Eier bezw. Junge, also mufs das Vorkommen von 6 Eiern sehr selten sein.

Turdus viscivorus $\mathrm{L}$. Nur vorübergehend beobachtet, hat hier nicht gebrütet.

Regulus regulus L. Besuchte öfter die Kiefernwälder, mufs in der Nachbarschaft gebrütet haben.

9. Phylloscopus rufus (Bchst.). Brutvogel im Parke öfter beobachtet.

10. Phylloscopus trochilus (L.). Brutvogel im Parke, am 4. 6. fand ich, sehr versteckt in altem Eichenlaub, ein Nest mit Jungen.

11. Hypolais hypolais (L.). 2 Paare nisteten im Parke, die Nester waren in $2 \mathrm{~m}$ Höhe in jungem Haselaufschlag eingeflochten und waren nur 30 Schritte von einander entfernt. Das eine Gelege war am 9.6. mit 5 E. vollzählig, das andere am 13. 6. 
11. Calamodus schoenobaenus (L.) u. Acrocephalus streperus (Vieill.). Beide Arten hielten sich vorübergehend an einem kleinen Weiher im Parke auf ohne jedoch zu brüten. Das Schilfrohr fehlte! -

12. Sylvia nisoria (Bchst.). Ein Paar im Parke, das Nest war am 25. 5. mit 5 Eiern belegt, es stand in etwa $1 \mathrm{~m}$ Höhe im Brombeergestrüpp in dichter Nachbarschaft (cr. $5 \mathrm{~m}$ ) eines Würgernestes (Lanius colluris). brütend.

13. Sylvia atricapilla (L.). Wohl nur ein Paar im Parke

14. Sylvia simplex (Lath.). Im Parke zu mehreren Paaren brütend, ich fand 2 Nester, eins stand in einer Koniferengruppe auf einer kleinen Fichte, nach Drosselart dicht am Stamme, es enthielt am 23. 5. 4 Eier; das andere stand im Himbeergesträuch und enthielt am 25. 5. 5 Eier.

15. Sylvia sylvia (L.). Die Dorn-Grasmücke, als häufigste Sylvia-Art Charaktervogel der Landschaft. Überall wo die zahlreichen Gräben, Feld- und Waldränder von Dornengestrüpp begrenzt werden wird man diesen Vogel antreffen. Ich fand sogar ein Nest unter einem Ginsterbusch in der Blöfse einer Kiefernschonung; ein anderes in einem isoliert stehenden, kaum gröfser als kopfgrofsen, Ulmenanschlag auf einem Grenzrain. Volle Gelege fand ich in der Zeit vom 21.5.-7. 6., sie enthielten zwei 5 und zwei 6 Eier.

16. Sylvia curruca (L.). Überall beobachtet, doch nicht so häufig wie die vorige.

\section{Troglodytes troglodytes (L.). Im Parke beobachtet.}

18. Parus major L. 2 Paare beobachtet. Schon seit Jahren wird ein gufseisernes Pumpenrohr auf dem Heidefriedhof als Niststätte benutzt. Im vergangenen Jahre mufste im Mai zwecks einer Reparatur der Deckel abgeschraubt werden, da fand man 14 halbflügge Junge vor. In diesem Jahre bestand die 1. Brut wieder aus 14 Jungen, die 2., die heute am 1. Juli bereits Flugund Sprungübungen macht, aus $6 \mathrm{Köpfen}$. Mit dieser Wahl der Pumpe als Niststätte haben die Meisen recht klug gehandelt, denn einmal ist diese eiserne Kinderstube gegen alle 2- und 4-beinigen Räuber sicher und dann müssen die Jungen auch schon recht kräftig sein, um überhaupt hinaus zu können, da die einzige Öffnung etwa $30 \mathrm{~cm}$ senkrecht über dem Neste ist. Diese ideale Meisenwohnung ist auch immer bewohnt, ein Zeichen ihrer Beliebtheit.

19. Parus ater L. Ein Paar im Kiefernstangenholz.

20. Parus cristatus L. Ein Paar Haubenmeisen nistete in einer alten hohlen Eiche. Dieser Baum stand am Waldrande 
auf einer breiten Trift und hatte in Mannshöhe ein altes Spechtloch, das im Laufe der Zeit so erweitert worden war, dafs ich mit der Hand hineinfassen konnte und zwar bis auf den $25 \mathrm{~cm}$ tiefen Grund. Da alte, hohle Bäume hier rar sind, hatte ich gleich vom 1. Tage meines Hierseins ein Augenmerk auf diese geeignete Niststätte, und zu meiner Freude wurde sie auch Mitte April von der Haubenmeise bezogen. Am 5. Mai war das Gelege mit 14 Eiern vollzählig, ich bereicherte hiermit meine Sammlung. Die alte Meise safs sehr fest auf den Eiern und war nur sehr schwer zum Verlassen des Nestes zu bewegen, näherte ich mich nur dem Baume, so zischte es mir aus der Öffunng heraus entgegen, klopfte ich aber an den Baum, so ging dieses Zischen in ein Fauchen und Keifen über, dafs man in der Höhlung eher eine Eule oder einen Iltis vermutete als ein kleines Meischen, wagte ich nun gar die Hand hinein zu stecken, so flogen mir ganze Wolken Haare, Moos und anderes Nistmaterial entgegen.

21. Aegithalus candatus (L.). Im Parke wurde im April das Nest vom Eichhörnchen zerstört, es befand sich in $2 \frac{1}{2} \mathrm{~m}$ Höhe am Stamme einer jungen Eiche, das 2. Nest wurde am anderen Ende des Parks in einer Koniferengruppe zwischen 2 dicht nebeneinander gewachsenen jungen Fichten gebaut, es war nur $1 \frac{1}{4} \mathrm{~m}$ über dem Erdboden, die Jungen flogen aus. Ein zweites Paar nistete in einer älteren, gemischtlaubigen Schonung.

Certhia familiaris L. Im April öfter beobachtet, hat hier jedoch nicht gebrütet.

22. Alauda arvensis L. Nach meinen Beobachtungen ist die Feldlerche die Vogelart, die in meinem Bezirke am zahlreichsten vertreten ist, sie ist auf Wiese und Feld gleich häufig. Ich fand allein auf einem 15 Morgen grofsen Haferfelde 3 belegte Nester, und zwar ohne danach zu suchen, sondern nur zufällig.

23. Lullula arborea (L.). In den jungen Kiefernanpflanzungen häufig. In der 2. Hälfte des April wurde bei den Kulturarbeiten ein Nest mit 5 Eiern gefunden.

24. Galerida cristata (L.). Weniger häufig beobachtet, ein Nest mit 3 Eiern fand ich am 17. 6. auf einer alten brach liegenden Mietenstelle, jedenfalls 2. Brut.

25. Budytes flavus (L.). Verhältnismäfsig häufig, häufiger als Mot. alba, indem von ersterer 4 Brutpaare, von letzterer nur 3 Brutpaare hier sind. Sie bevorzugt so wie kein anderer Vogel als Niststätte die Grabenränder, ich fand alle 4 Nester an solchen und zwar immer sehr geschickt und gut versteckt angelegt. In einem Neste fand ich schon am 24. 5. Junge, also sind die Gelege mitunter schon vor Mitte Mai vollzählig. 2 Gelege wurden geräubert, ich weifs nicht, auf wessen Konto ich diese Untat schreiben soll. In dem einen Falle war das Nest mit 
herausgerissen. Dieses soll ja eine Eigentümlichkeit der Eulen sein.

26. Motacilla alba L. Unter dem Firste eines halb verfallenen Ziegelschuppens entdeckte ich am 19.5. ein Bachstelzennest mit 2 Eiern, 3 Tage später entnahm ich dem Neste 4 Bachstelzeneier und 1 Kuckucksei, das 5. Bachstelzenei hat der Kuckuck jedenfalls entfernt. Spuren davon konnte ich allerdings nicht entdecken.

27. Anthus pratensis (L.). Bevölkern gemeinsam mit den Feldlerchen die Wiesen, dort aufserordentlich häufig. Die Jungen haben, ähnlich so wie die Lerchen, sehr lange Dunen und sind dadurch ihrer Umgebung vorzüglich angepafst. Am zahlreichsten waren sie in der Wiese, dort, wo die Sauergräser, als Seggen, Binsen standen; infolge der Trockenheit waren diese Stellen am kahlsten in diesem Jahre.

28. Anthus trivialis (L.). An den Waldrändern zahlreich; mir fiel auf, dafs sie die Ostseite der Baumgruppen und Waldparzellen bevorzugen.

29. Anthus campestris. Im Mai beobachtete ich öfter den Brachpieper in der Nähe einer gröfseren Sandgrube, die von Unland umgeben war, ich zähle ihn deshalb mit zu den Brutvögeln meines Bezirks.

30. Miliaria calandra (L.). Überall anzutreffen, ein häufiger Vogel.

31. Emberiza citrinella L. Recht häufig, nistet überall, sowohl in der Wiese als auch in der Kiefernschonung. Am 4. 6. fand ich im Parke auf einer kleinen Fichte ein Nest mit 5 Eiern, die durch ihre anormale schlanke Form interessant sind, während nach Friedrich der Durchschnitt von 85 Eiern 21,3 $\times$ $15,9 \mathrm{~mm}(4: 3)$ beträgt, war das Durchschnittsmafs meines Geleges $23 \times 15,5 \mathrm{~mm}(3: 2)$.

32. Emberiza hortulana L. Der Ortolan brütet hier in mindestens 8 Paaren, ist also relativ recht gemein. Er ist mehr ein Vogel des freien Feldes, wenn nur genügend mit Bäumen bepflanzte Wege dasselbe durchschneiden. Das Nest stand in keinem Falle am Wege oder Grabenrande, sondern im Getreidefelde; es wurde mir erst durch Futter tragende Alte verraten.

33. Acanthis cannabina (L.). Beobachtete nur 1 Brutpaar. Das Nest stand in einer Kiefernschonung und enthielt am 5. 5 . 4 Eier.

34. Chloris chloris (L.). In einer Fichtengruppe im Park 2 Nester. Die Jungen waren bereits Anfang Mai flügge.

35. Fringilla coelebs L. Im Walde und Parke der häufigste Vogel, auch überall in den gröfseren Kiefernschonungen, die 
zahlreich mit Birken durchsetzt sind. Im Parke fand ich mehrere Nester in den Astgabeln junger Bäume und Sträucher; eins stand nur in reichlich Manneshöhe in einem Holunderbusch. Vollzählige Gelege fand ich vom 28. 4.-9. 5., sie enthielten meist 5 Eier.

36. Passer domesticus (L.). Der Haussperling nicht in so grofser Zahl vorhanden, wie das häufig der Fall zu sein pflegt. Seit Wochen betätigt er sich übrigens sehr nützlich in den nahen Kiefern-Schonungen beim Raupenfang, und zwar ist es die in diesem Jahre sehr zahlreich auftretende Kiefern-Eule (Trachea pimiperax), der er nachstellt. Auch die Stare helfen eifrig bei dieser Arbeit.

37. Passer montanus (L.). In einer hohlen Pappel hinterm Gehöfte mehrere Paare.

38. Sturnus vulgaris L. Im Parke sehr zahlreich, besonders in den aufgehängten Nistkästen.

39. Oriolus oriolus (L.). Mit 3 Brutpaaren in diesem Bezirk ist der Pirol als relativ häufig zu bezeichnen. Die Nester standen im Kiefernstangenholze in etwa 10-12 $\mathrm{m}$ Höhe; am 4. 6. enthielt das eine 3 schwach bebrütete Eier. Im Parke war der Pirol stets anzutreffen, nistete dort aber nicht.

40. Garrulus glandarius (L.). Vor der Brutzeit trieb sich der Eichelhäher in Scharen von 5-7 Stück überall herum; zur Brut sind nur 2 Paar geschritten, die andern strichen weiter.

41. Pica pica (L.). Ein Nest entdeckt in einer älteren Schonung, es stand in ca. $5 \mathrm{~m}$ Höhe. Am 15. 5. waren die Jungen bereits ausgeflogen.

42. Corvus cornix L. Hier brüteten 7 Paare, 2 auf Birken und 5 auf Kiefern, alle Krähen wurden abgeschossen, da ich in ihnen die gefährlichsten Nesträuber unter den Vögeln kennen gelernt habe, besonders haben sie es auf die bodenständigen Nester abgesehen und kein Rebhuhn-, Kiebitz- und Fasanengelege ist sicher vor ihnen. In einer Grenzschonung beobachtete ich öfter 1 Paar: 1 Elternvogel cornix und 1 Elternvogel corone.

Corvus frugilegus L. Im zeitigen Frühjahr in grofsen Scharen auftretend habe ich sie später hier nicht mehr gesehen, in der näheren Umgebung der Kreisstadt Herzberg (Elster) sollen umfangreiche Kolonien sein.

43. Lanius excubitor L. An geeigneten Stellen hier überall anzutreffen, in meinem Gebiet nisteten 2 Paare. Die Nester standen im Stangenholze auf den äufsersten Wipfeln junger Kiefern in $8 \mathrm{~m}$ und $10 \mathrm{~m}$ Höhe. Die Nistbäume waren nur durch eine ca. $300 \mathrm{~m}$ breite Blöfse getrennt. Ich beobachtete den Raubwürger im April täglich, wie er rüttelnd über den jungen Anpflanzungen und Blöfsen dem Käferfange oblag, und zwar fielen ihm meist die nützlichen 
Laufkäfer zur Beute, ich beobachtete ihn aber auch, wie er mit Ausdauer und Mordlust den Finken und Meisen nachstellte. Der Mageninhalt zweierVögel, die Ende A pril geschossen wurden, bestand nur aus Käferresten, Knochenreste dagegen fand ich in dem Magen eines Weibchen, das am 5. V. vom Neste geschossen wurde, nachdem 30 Stunden znvor dem Neste 7 schwach bebrütete Eier entnommen waren. Ich habe bei diesem Würger als auch bei seinem Vetter collurio die Beobachtung gemacht, dafs beim $ᄋ$ der Haken des Schnabels stärker ausgebildet ist als beim $\sigma^{x}$. Bei meinen verhältnismäfsig wenigen Beobachtungen wage ich nicht dieses als bewiesene Tatsache aufzustellen.

44. Lanius collurio L. Überall eine häufige Erscheinung, bevorzugt als Niststätte die Brombeergestrüppe an den Grabenund Wegerändern, sein sonst so beliebter Schlehdorn gedeiht hier nicht, da der Boden zu leicht ist. Im Parke ist das Nest häufig in den mit Hopfen umrankten Hasel- und Erlensträuchern zu finden. In der Zeit vom 26. V.-9. VI. fand ich 9 volle Gelege, darunter 2 mit je 7 Eiern. Die Eier eines Geleges sind in Färbung und Gestalt sehr ähnlich, die verschiedenen Gelege aber weichen erheblich voneinander $a b$, so dafs ich, die Eier der 9 Gelege bunt durcheinander gemengt, ohne Mühe die zusammengehörigen Eier herausfinde. Der Würger hängt mit einer bewunderungswürdigen Zähigkeit an seinem Neste und ist so leicht nicht $\mathrm{zu}$ bewegen, dasselbe im Stiche $\mathrm{zu}$ lassen, ich habe ihm haselnufsgrofse Kiesel ins Nest gepackt, das \& täglich 10 und mehr mal vom Neste gejagt, aber immer kehrte es bald wieder zurück und brachte auch die Jungen aus. Ich möchte bei dieser Gelegenheit es nicht unerwähnt lassen, dafs ich es für eine Mär halte, die Vögel verliefsen ein von Menschenhand berührtes Gelege, bei den Kleinvögeln jedenfalls trifft dieses nur im Ausnahmefalle $\mathrm{zu}$, dagegen sind z. B. die Tauben, sowohl palumbus als auch turtur, aufserordentlich empfindlich, und ich habe wiederholt beobachtet, dafs sie, vom Neste gejagt, selbst Junge im Stich liefsen und dem Hungertode preisgaben.

45. Muscicapa grisola L. 3 Paare beobachtet immer in Nähe von Geländen, ein Nest stand, nach Finkenart, auf dem wagerechten Aste einer alten Kastanie.

46. Hirundo rustica L. Im ganzen Dorfe zählte ich 43 besetzte Nester und zwar 15 auf dem Gute und 28 bei den 7 Stellenbesitzern. Heute am 3. Juli haben die meisten bereits mit der 2. Brut begonnen. Bemerkenswert ist, dafs die Schwalben zur 2. Brut mitunter einen Wohnungswechsel vornehmen, so erzählte mir ein Bauer hier, dafs bei ihm ein Schwalbenpärchen schon seit 3 Jahren stets zum 1. mal im Kuhstalle, zum 2. mal im Pferdestalle brütete. Einen Nestwechsel in demselben Stalle habe ich öfter beobachtet. Im Hühnerstalle benützen die Schwalben zum Ein- und Ausfliegen das am Erdboden befindliche Hühnerloch. 
47. Chelidonaria urbica (L.). 17 besetzte Nester im Orte und zwar in 4 kleinen Kolonien brütend.

Apus apus (L.). Vereinzelt beobachtet, jedenfalls aus dem $15 \mathrm{~km}$ entfernten Herzberg.

48. Caprimulgus europaeus L. Im Walde 1 Paar, hält sich in einer zwischen 2 Gehölzen liegenden, älteren Schonung auf. Trotz eifrigen Suchens konnte ich das Gelege nicht entdecken. Das Männchen liefs sein abendiches Geknarre besonders von 3 Lieblingsbäumen hören und zwar immer von demselben Aste. An geeigneten Stellen der Umgebung überall anzutreffen. Kommt allabendlich in den Park zum Insektenfange.

49. Upupa epops L. Ist in dieser Gegend eine ständige Erscheinung. In meinem Revier brüteten 2 Paare, Ankunftsdatum in diesem Jahre war der 3. April. Von Mitte April ab hörte ich den Paarungsruf zuerst nur morgens und abends, dann im Mai zu jeder Tageszeit. Ähnlich wie die Nachtschwalbe hat auch der Wiedehopf seine Lieblingsplätze, von denen aus er sein „hup-hup-hup“ erschallen lärst, das von einem ständigen Auf- und Abschlagen des Federbusches begleitet ist, wird der W. hierbei nicht gestört, so kann er wohl l Stunde auf ein und derselben Stelle aushalten. In einem Gehölze grenzten die Brutbezirke der beiden Paare, trafen sich zufällig dort die Männchen, so gab es erbitterte Kämpfe, denen man trotz allen Gefauches, Grimasseschneidens und Aufblähens anmerkte, wie beide gegenseitig voreinander Furcht hatten und stets in respektvoller Entfernung so zu sagen „3 Schritte vom Leibe“ blieben. Am 21. V. fand ich in derselben Höhlung, der ich am 5. V. das Gelege der Haubenmeise entnommen hatte, das $\subsetneq$ auf 4 Eiern sitzend, da es durch nichts zu bewegen war, das Gelege zu verlassen, holte ich es mit der Hand heraus, was es sich ohne die geringste Gegenwehr gefallen liefs, erst als ich es im Freien hatte, sträubte es sich plötzlich und büfste hierbei leider einen Teil der Schwanzfedern ein. Da ich am nächsten Tage das Gelege verlassen und nicht vermehrt fand, entnahm ich es für meine Sammlung. In den folgenden 4 Tagen revidierte ich noch mehrere Male ohne Erfolg die Höhlung und besuchte sie dann erst wieder am 5. VI., wer beschreibt mein Erstaunen, als ich wieder den Wiedehopf auf 7 Eiern sitzend im Neste fand. Dafs es dasselbe $q$ war, unterliegt kaum einem Zweifel, da das andere Paar, das in Frage kommen könnte, zu dieser Zeit schon Junge hatte, die am 24. VI. schon ausg eflogen sind. Es mufste also das ${ }_{\text {, }}$, vielleicht infolge eines Choks, vor Angst und Schreck, das Legen 5-6 Tage eingestellt haben. Dem Gelege raubte ich noch 3 völlig unbebrütete Eier, die bleibenden 4 brütete das $\$$ aus, und ich habe heute, am 3. Juli, die Jungen, als die ersten ihrer Art, für die Helgoländer Vogelwarte beringt. 
50. Cuculus canorus L. Seine Ankunft fiel hier auf den 24. April. Es trieben sich häufig 2 und mehr Männchen herum, die sich dann eifrig befehdeten. Am 22. V. fand ich ein Bachstelzengelege (Motacilla alba) mit Kuckucksei.

51. Picis viridis L. Der einzige Specht, der in meinem Bezirke Brutvogel ist, und zwar brütete er im Parke in einer Eiche, ca. $3 \mathrm{~m}$ überm Erdboden.

Dryocopus martius (L.). 1 Männchen besuchte den ganzen April hindurch bis Ende Mai tagtäglich die alten Kiefernstubben in den Schonungen. Sein Kommen kündete er stets durch das weitschallende ,krick krick krick“ an; nach längerer Arbeit an den Stubben suchte er dann auch einen Baum auf und liefs das lang gezogene „,kliöh“ hören. Da er gerade nicht zu den Fliegern von Gottes Gnaden gehört, glaube ich mich zu der Annahme berechtigt, dafs sein Nistbaum in nicht allzu weiter Entfernung gestanden hat.

Jynx torquilla L. In den ersten Tagen des Mai hielt sich hier mehrere Tage ein Wendehals auf, der dann aber weiterzog.

52. Athene noctua (Retz). In einer hohlen Weide am Gartenrande nistete 1 Paar.

53. Asio otus (L.). Am 4. 5. klopfte ich aus einem alten Krähenneste im Kiefernstangenholze 1 Waldohreule heraus. Am 7. 5. untersuchte ich das Nest und fand 2 frisch gelegte Eier.

Buteo buteo (L.). Wunderbarerweise ist kein Raubvogel in meinem Bezirke Brutvogel. Der Mäusebussard ist in dieser Gegend nicht so häufig, wie es z. B. in der Mark der Fall ist. Ein Paar war häufig hier zu sehen, mufs in nicht allzuweiter Entfernung gebrütet haben.

Falco subbuteo L. Kam öfter und beunrubigte die Schwalben, ich habe hierbei die Eleganz, den Schneid und die Gewandheit dieses kühnen Fliegers bewundern können. Einmal bot sich mir sogar die Gelegenheit, seine Schnelligkeit zu kontrollieren. Ich befand mich auf der Wiese bei Heuarbeiten, als ich einen Baumfalken heranstreichen sah. Er flog so nahe an mir vorbei, dafs ich mit blofsem Auge die schwarzen Backenstreifen erkennen konnte. Als er auf der anderen Seite der Wiese das Feld erreicht hatte, machte er eine fast rechtwinkelige Schwenkung und jagte nun in rasender Fahrt längs eines Weges über die Felder dahin. Ich hatte gleich bei der Schwenkung die Uhr gezogen und zählte bis zum Walde 8 Sek. Die Strecke war 620 Schritt lang, etwa $460 \mathrm{~m}$. Dieses würde eine Stundengeschwindigkeit von weit über $200 \mathrm{~km}$ ausmachen.

Astur palumbarius (L.). Einmal im Mai sah ich den Hühnerhabicht über einer Schonung dahinjagend. 
Accipiter nisus (L.). Öfter beobachtet, nicht so häufig wie in der Mark.

Circus pygargus (L.). Im April öfter auf dem Durchzuge beobachtet.

54. Turtur turtur (L.). Fin Paar Brutvögel im Parke, ein anderes an einer geeigneten Stelle in einem Stück Mischwald.

55. Columba palumbus L. Überall eine häufige Erscheinung, trotz seiner Häufigkeit und trotzdem ihm nicht im geringsten nachgestellt wird ein sehr scheuer Vogel. Im Parke fand ich am 3. Juli ein Nest mit wenige Tage alten Jungen im wilden Hopfen, etwa $3 \mathrm{~m}$ über dem Erdboden.

56. Perdix perdix (L.). Zahlreich vertreten, am 20. 5. fand ich bereits ein Gelege mit 17 Eiern. Sehr viele Gelege haben die Krähen zerstört, besonders in den Wiesen.

57. Coturnix coturnix (L.). Hörte den Lockruf nur $1{ }^{7}$, in einer $25 \mathrm{Mrg}$. grofsen Haferparzelle in der Wiese.

58. Phasianus colchicus L. Seit einigen Jahren erst künstlich angesiedelt.

Tetrao tetrix L. Auf unseren Wiesen war ein Balzplatz, auf dem die Birkhühner der ganzen Umgegend zusammen kamen. Bis Mitte Mai habe ich die Birkhühner in meinem Revier beobachtet, dann waren sie verschwunden, und keine Henne brütete bei mir, trotzdem es an geeigneten Heideflächen nicht mangelt, vielleicht sind diese nicht grofs genug.

Otis tarda L. Früher, noch vor 12 Jahren, hier in Grauwinkel Brutvogel, aber seitdem durch den Zwischenfruchtbau die Brachbaltung abgelöst wurde, hat sich die Trappe mehr ins Brandenburgische verzogen, dort in dem 1 Meile von hier entfernten Bärwalde soll sie im Winter in Scharen bis zu 70 Stück auf den Rapsfeldern zu sehen sein.

Ciconia ciconia (L.). Auch hier ist der Storch im Abnehmen begriffen, und er ist jetzt eine beinahe seltene Erscheinung geworden. Bis vor einigen Jahren (in Grauwinkel 5 Jahren) befanden sich hier im Orte und in allen Nachbardörfern, 5 an der Zahl, je 1 besetztes Storchnest, jetzt sind alle verlassen; das nächste besetzte Nest befindet sich in dem $8 \mathrm{~km}$ entfernten Bernsdorf. Die Ursache der Abnahme des Storches vermag ich nicht zu ergründen, Mangel an geeigneten Nistgelegenheiten ist es nicht, denn hier gibt es noch Strohdächer in Hülle und Fülle.

59. Vanellus vanellus (L.). In den Wiesen 4 Brutpaare.

60. Oedicnemus oedicnemus (L.). In den ausgedehnten Kiefernanpflanzungen ein Brutpaar. Zur Brut sind die Vögel bis jetzt (3. VII.) noch nicht geschritten, oder aber das Gelege ist ein Opfer der Krähen geworden. Der Triel ist in dieser 
Gegend nicht selten, ich habe ihn wiederholt in der Nachbarschaft gesehen und nachtsauchs rufen hören.

61. Anas boschas L. Die Stockente ist hier nicht sehr häufig, wohl infolge Mangels geeigneter Gewässer, grölsere Wasserflächen gibt es garnicht und auch kleinere sind sehr selten. Iu der Umgebung des nur einige $100 \mathrm{qm}$ grofsen Weihers im Parke haben 2 을 gebrütet. Ende Juni sah ich die beiden Schofe, während der eine vollkommen flügge war, bestand der andere aus Entlein, die erst vor wenigen Tagen aus dem Ei gekrochen waren.

62. Colymbus fluviatilis Tunst. In dem kleinen Weiher im Parke alljährlich ein Brutpaar.

\section{Zur Biologie der Zwergrohrdommel (Ardetta minuta).}

\section{Von Friedrioh von Luoanus.}

(Hierzu Tafel 1 und 2.)

Das Leben der Vögel, vor allem ihre intimeren sitten und Gewohnheiten draufsen in der Natur zu beobachten, ist häufig recht schwierig, besonders wenn es sich um Vogelarten handelt, die Bewohner unzugänglicher Orte, des Sumpfes und der Rohrlandschaft sind.

Das beste Mittel das Leben und Treiben solcher Vögel kennen zu lernen, besteht darin, dafs man junge Vögel aus dem Nest nimmt und aufzieht. Man erhält so wirklich zahme Tiere, die durch die Gegenwart des Menschen in keiner Weise sich stören lassen, all ihre Gewohnheiten ohne jede Scheu verrichten und so die beste Gelegenheit für biologische Studien geben. Der Einwand, dafs der von Menschenhand aufgezogene Vogel viel von seiner Natürlichkeit einbüfst, und dafs man daher aus seinem Verhalten keine Schlüsse auf das Freileben ableiten kann, ist nicht stichhaltig. Bei dem Tier und besonders bei dem Vogel spielt nun einmal das Reflektorische eine grofse Rolle. Die Art der Nahrungsaufnahme, die verschiedenen Stellungen und Gebärden, die Balz der Männchen, alles dies sind angeborene Triebe und Instinkte, die der jung aufgefütterte, zahme Vogel in der Gefangenschaft in derselben Weise äufsert, wie der wilde Vogel in der Freiheit.

Ganz besonders wertvoll ist die Aufzucht junger Vögel für psychologische Beobachtungen. Nur an einem zahmen Tier, das seinem Pfleger mit völligem Vertrauen begegnet, kann man das Seelenleben nach den verschiedensten Richtungen hin prüfen, aber nicht an einem scheuen Wildling, der beim Anblick des Menschen nur immer unter dem Banne des Fluchtreflexes steht. 

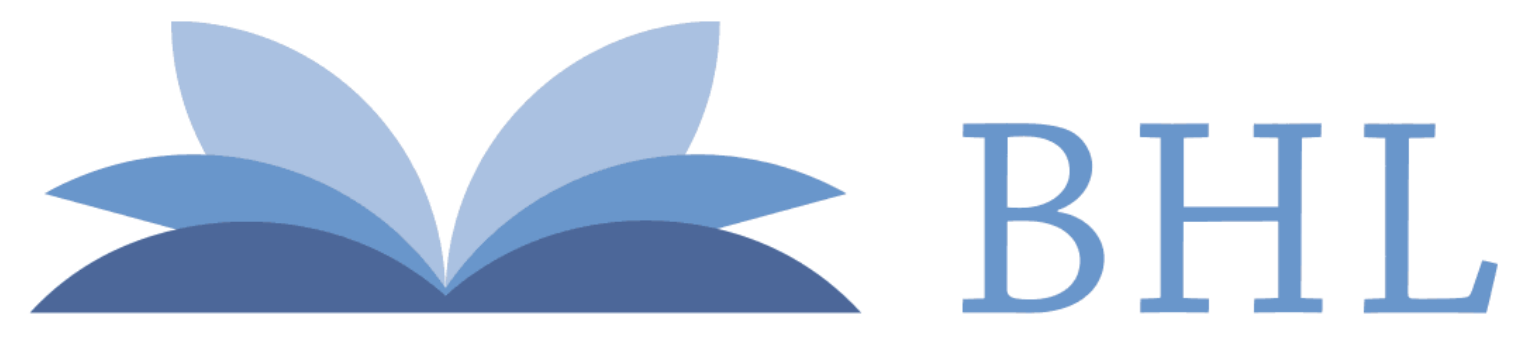

\section{Biodiversity Heritage Library}

1914. "Ein kleiner Beitrag zur Kenntnis der Ornis der Provinz Sachsen." Journal fu

r Ornithologie 62, 39-49. https://doi.org/10.1007/bf02089370.

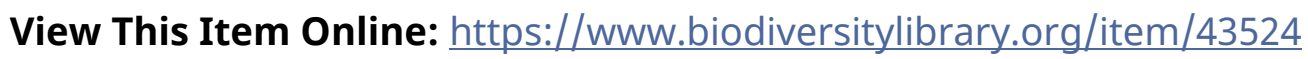

DOI: https://doi.org/10.1007/bf02089370

Permalink: https://www.biodiversitylibrary.org/partpdf/142778

\section{Holding Institution}

MBLWHOI Library

\section{Sponsored by}

MBLWHOI Library

\section{Copyright \& Reuse}

Copyright Status: No known copyright restrictions as determined by scanning institution.

This document was created from content at the Biodiversity Heritage Library, the world's largest open access digital library for biodiversity literature and archives. Visit BHL at https://www.biodiversitylibrary.org. 